JOURNAL of

MAINE MEDICAL CENTER Journal of Maine Medical Center

\title{
Focused Hospital Medicine CME on Pulmonary Point-of-Care Ultrasound through Just-in-Time Training During the COVID-19 Pandemic
}

\author{
Matthew Buttarazzi \\ Maine Medical Center
}

Et al.

Follow this and additional works at: https://knowledgeconnection.mainehealth.org/jmmc

Part of the Internal Medicine Commons, and the Interprofessional Education Commons

\section{Recommended Citation}

Buttarazzi, Matthew; Bartlett, Kristen; Stebbins, Katherine; and Thim, Monica (2021) "Focused Hospital Medicine CME on Pulmonary Point-of-Care Ultrasound through Just-in-Time Training During the COVID-19 Pandemic," Journal of Maine Medical Center. Vol. 3 : Iss. 1 , Article 10.

Available at: https://knowledgeconnection.mainehealth.org/jmmc/vol3/iss1/10 https://doi.org/10.46804/ 2641-2225.1072

The views and thoughts expressed in this manuscript belong solely to the author[s] and do not reflect the opinions of the Journal of Maine Medical Center or MaineHealth.

This Innovation Highlight is brought to you for free and open access by Maine Medical Center Department of Medical Education. It has been accepted for inclusion in the Journal of Maine Medical Center by an authorized editor of the MaineHealth Knowledge Connection. For more information, please contact Dina McKelvy mckeld1@mmc.org.

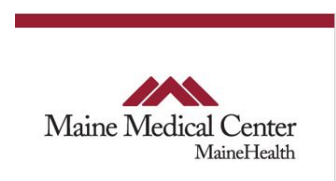


Focused Hospital Medicine CME on Pulmonary Point-of-Care Ultrasound through Just-in-Time Training During the COVID-19 Pandemic

\section{Acknowledgements}

Funding provided by the Maine Medical Center Adult Medicine Service Line and a Department of Medical Education educational initiative.

Authors

Matthew Buttarazzi, Kristen Bartlett, Katherine Stebbins, and Monica Thim 


\title{
Focused Hospital Medicine CME on Pulmonary Point-of- Care Ultrasound through Just-in-Time Training During the COVID-19 Pandemic
}

\author{
Matt Buttarazzi MD, Kristen Bartlett MD, Katherine Stebbins PA-C, Monica Thim DO \\ Department of Medicine, Maine Medical Center, Portland, ME
}

\begin{abstract}
Introduction: We describe the use of focused CME with just-in-time training in pulmonary point-of-care ultrasound (POCUS) for hospitalists as part of our response to coronavirus disease 2019 (COVID-19). This training served as a pilot for developing a longitudinal training program for our inpatient adult medicine providers.
Methods: In mid-March 2020, ultrasound education and image review were deployed within hospital medicine at a large academic medical center. Faculty were provided (1) a small-group lecture focused on ultrasound findings in pneumonia, (2) weekly review of imaging, and (3) a monthly review conference of ultrasound imaging.
\end{abstract}

Results: $\quad$ Use of POCUS for pulmonary pathology at our institution increased at the start of the pandemic. Providers eligible for privileging increased from $4 \%(2 / 45)$ to $20 \%(9 / 45)$ within 1 month of undergoing ultrasound education. Use of devices continued for 3 months after the last training was conducted.

Discussion: Just-in-time training in pulmonary ultrasound incentivized by the COVID-19 pandemic provided an excellent pilot test for developing a longitudinal CME program focused on POCUS education for hospital medicine. This project demonstrated the need for clear privileging guidelines for physicians and advanced practice professionals.

Conclusions: Just-in-time training in pulmonary ultrasound was well received by a large hospital medicine group and increased ultrasound use during the COVID-19 pandemic.

Keywords: $\quad$ POCUS, COVID-19, Just-in-Time, credentialing, hospitalist

T he Society for Hospital Medicine leads national support for the development of programs to train hospital medicine in pointof-care ultrasound (POCUS). This effort has progressed significantly over the past few years. ${ }^{1,2}$ POCUS is used to evaluate many diseases managed by hospitalists. ${ }^{3-6}$ Additional benefits include shared understanding between providers and patients regarding diagnosis when ultrasound is used. ${ }^{7}$ However, the Accreditation Council for Graduate Medical Education does not require POCUS training in internal medicine residency, so education in POCUS remains variable at

Correspondence: Matt Buttarazzi, MD

Department of Medicine, Maine Medical Center

22 Bramhall St. Portland, ME 04102

BUTTAM@mmc.org the graduate education level. ${ }^{8,9}$ Publications of surveys demonstrate that incorporation is possibly increasing. A 2012 survey reported that 45 of 167 $(25 \%)$ internal medicine training programs included formal curriculum. ${ }^{9}$ In a 2018 survey, only 24 of $64(37.5 \%)$ internal medicine training programs included formal ultrasound training, suggesting that most practicing hospitalists acquired this skill after residency. ${ }^{10}$

There are no national standards for POCUS credentialing and privileging within hospital medicine or internal medicine societies. There are multiple external certificates and styles of effective training programs available. ${ }^{11,12}$ At our institution, privileging requirements were updated at the start of 2020 to include ultrasound for the first time. These requirements include completing $\mathrm{CME}$ and logging 
performed ultrasounds. Ten entries are required for each type of ultrasound exam requested.

There are many challenges to implementing POCUS into daily practice. Providers can participate in isolated, short-term training sessions. However, an inability to incorporate skills into daily practice limits their retention. ${ }^{13}$ Mathews et al hypothesized that integration into practice is limited by lack of device access and sustained time for honing techniquewhen learners complete CME in POCUS. Longitudinal models have had favorable outcomes for increasing skill retention in both faculty and residents. These models demonstrated skill growth proportional to each additional longitudinal session. ${ }^{13-15}$ Funding to support longitudinal ultrasound practice by faculty and resident ultrasound learners has been proposed but remains lacking at our institution. As a result of these barriers, only a small number of physicians meet standards for pulmonary ultrasound privileges at our medical center, despite completing the appropriate CME for privileging eligibility.

The coronavirus disease 2019 (COVID-19) pandemic presented many challenges-and opportunities-to health care systems. At our institution, patients who were ruled out for COVID-19 were cared for in powered air purifying respirators (PAPRs) as much as possible. The difficulty of PAPRs in the diagnostic exam is well known, as auscultation with a standard stethoscope is not possible. The utility of ultrasound was identified early in the preparation for the virus, with rapidly evolving literature in support thereafter. Early in the pandemic, reports from China revealed that COVID-19 had similar ultrasound presentations to previously described viral pneumonias. ${ }^{16,17}$

Preparing for the virus offered several opportunities to our group. This manifested primarily as increased time because the hospital census lowered when elective activities were stopped to comply with state regulations. The average census for the hospitalist team decreased $43 \%$, from 15 patients per team in the prior 4 months to 8.5 patients in April. This decrease provided time for staff to complete justin-time training and begin deliberate practice of ultrasound skills. In this paper we will describe the use of just-in-time training in pulmonary POCUS as part of our response to coronavirus disease COVID-19.

\section{METHODS}

At our large academic medical center, hospital medicine faculty with privileges in ultrasound developed a high-yield refresher training in pulmonary ultrasound that focused on pneumonia. This training was designed for our inpatient adult medicine physicians and advanced practice providers (APPs). The training was approved for CME credit at our institution, which holds accreditation status with the Joint Accreditation for Interprofessional Continuing Education. Lecture content included lung ultrasound basics: lung sliding, a-lines, b-lines, subpleural and larger consolidations, pleural effusions, and case review specific to COVID-19 patients. Logistical topics were also featured, including image archiving, documentation, and disinfection. Disinfection of the device and system was covered in detail and reviewed on multiple occasions. After the lecture, participants practiced on each other for 10 to 30 minutes. Several other formats of review material were vetted and distributed, such as instructional videos, lectures, and podcasts. Additionally, a recorded version of the lecture was produced for future reference and dissemination to other hospitalist programs within our health system, including the internal medicine and family medicine residencies.

Early in the pandemic, the training was first delivered to providers starting on COVID-19 care teams, with lectures given near the start of shifts. Many providers started scanning patients that day. A content expert was available to give the lecture nearly every day: a total of 11 times over 27 days as different providers rotated on service.

Portable ultrasound devices were provided by department funding and intramural support. Adult medicine inpatient teams, including COVID-19 care teams, rounded with these devices, which had access to image archiving and enabled review of images at care delivery by our department ultrasound faculty. Physicians and APPs began maintaining a log of studies to submit for privileging. Images were reviewed using previously described scoring rubrics. ${ }^{18}$ 
Physicians and APPs seeking to maintain future privileging participated in case and image review every 2 weeks during the height of the pandemic. The goal attendance was $50 \%$. During these conferences, the group was updated on any literature relevant to lung ultrasound in COVID-19. During each session, attendees reviewed 3 cases focusing on common pulmonary ultrasound findings. The remaining time was spent reviewing images taken by the providers in attendance. These image review conferences occurred monthly as the volume of COVID-19 cases stabilized.

\section{RESULTS}

Over 4 a week period, $51 \%$ (23/45) of physicians and $43 \%(13 / 30)$ of APPs completed the training. The recorded version of the lecture had 60 unique views. In the first 26 days following the last training, 3 or more providers signed out devices on $46 \%$ of the days (12/26). In the first 3 months after the initial 4 week period this trend continued, with 3 or more devices signed out on $49 \%$ of the days (45/91). Sign-out data also indicated ultrasound use for patients in other medical units. Specifically, devices were signed out $35 \%$ of the shifts $(91 / 260)$ by providers covering the COVID-19 units and $65 \%$ $(169 / 260)$ of the time by providers covering other medical units.

The only historical data available to our group regarding ultrasound use are images saved into the electronic medical record with an order. In the year before the pandemic, ultrasound images on only 10 patients were saved in this fashion, less than 1 patient per month. During the first month following their training, 8 providers saved a total of 195 pulmonary ultrasound images for review, representing 64 different patients. The number of patients per provider ranged from 2 to 10 (median: 5.5). Far more images were saved compared to the prior system.

Informal post-education surveys demonstrated positive effects on group engagement in ultrasound, including support to continue monthly sessions and further educational opportunities. All respondents who participated in the training reported benefit in preparing for the pandemic. Also, 65\% (13/20) of respondents agreed that "ultrasound adds value to my exam in a PAPR."

Before COVID-19, only 4\% (2/45) of providers were privileged in POCUS. Four providers obtained the image numbers needed to resubmit privileging for pulmonary ultrasound within 2 weeks of completing the CME content. An additional 3 providers completed within 4 weeks. In total, $20 \%$ (9/45) of providers met requirements after the education period. In the 4 weeks after the lecture, 3 APPs saved 49 pulmonary images across 11 patients. One APP would have qualified for privileging under the physician guidelines; however, no local guidelines currently exist for APP privileging in ultrasound.

\section{DISCUSSION}

The COVID-19 pandemic provided opportunities to increase ultrasound use in a large hospitalist group. Providers were able to complete just-in-time training in pulmonary ultrasound and then obtain the image portfolios needed to seek privileging in pulmonary ultrasound in the department of medicine. We did not find evidence in the literature that similar programs were deployed at other institutions during the pandemic.

There are multiple limitations to the data we collected during the training implementation. The available historical data was limited to only ultrasounds saved in the electronic health record. These data are unlikely to represent how often devices were used due to multiple barriers for image retention in the system before COVID-19. Therefore, we cannot conclude if the training influenced ultrasound use or was a result of the pandemic alone. Additionally, increased access to more portable ultrasounds may also increase ultrasound use.

This just-in-time training program highlighted multiple areas for future work and improvement within POCUS education for our hospitalist group. APP attendance and subsequent use of ultrasound highlighted the need for consensus of privileging and supervision standards for our APPs interested in POCUS. This need prompted us to develop an ultrasound lead for the APP group and to add APP education, credentialing, and privileging to the ongoing education project. Expansion of ultrasound under COVID-19 funding included adopting a system with basic quality assurance functions, including imaging review to support privileging and monthly POCUS conferences.

The gap between the number of devices signed out and the number of saved patients per day warrants further investigation. We cannot ascertain if providers are not saving their images or carrying 
Journal of Maine Medical Center, Vol. 3 [2021], Iss. 1, Art. 10

a machine for the day without use. The former may be more likely, as the informal post-survey showed that more faculty reported a positive effect of ultrasound in managing patients under isolation precautions than the total providers who saved images. Further assessment of this program and future POCUS CME will include whether using other diagnostic modalities (eg, chest x-ray) decreased, and whether provider confidence was affected by this training. As more providers become skilled in ultrasound, we hope to see significant changes in undergraduate and graduate medical education, not only in crossover of content created for faculty training, but also in the efficacy of the education.

\section{CONCLUSIONS}

For several years, our hospitalist group made slow progress in ultrasound use due to multiple barriers. As with many issues in health care, these barriers relate to time and cost. The COVID-19 pandemic has stressed the health care system. However, the pandemic also temporarily decreased volume at our hospital, enabling physicians to focus on integrating ultrasound into daily practice. The implementation of just-in-time training and CME served as a successful pilot test of a faculty development program and was well received by trainees. A formal quality assurance program will be imperative as more providers meet privileging standards. Sustaining and expanding such a program will require significant effort and institutional resources. In the future, we will have many opportunities to study the implementation and effects on patient care as well as the long-term benefits to residents, students, and APPs. Overall, this just-in-time training has accelerated ultrasound use for our hospitalist group and will serve as the foundation for future multidisciplinary work within our department, hospital, and health care system.

Acknowledgements: Funding provided by the Maine Medical Center Adult Medicine Service Line and a Department of Medical Education educational initiative.

\section{Conflict of Interest: None}

\section{REFERENCES}

1. Soni NJ, Schnobrich D, Mathews BK, et al. Point-of-care ultrasound for hospitalists: a position statement of the Society of Hospital Medicine. J Hosp Med. 2019;14:E1-E6. doi:10.12788/ jhm.3079

2. Lucas BP, Tierney DM, Jensen TP, et al. Credentialing of Hospitalists in ultrasound-guided bedside procedures: a position statement of the Society of Hospital Medicine. J Hosp Med.

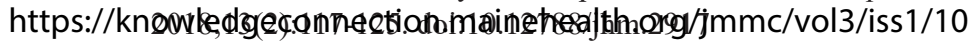
DOI: $10.46804 / 2641-2225.1072$
3. Soni NJ, Franco R, Velez MI, et al. Ultrasound in the diagnosis and management of pleural effusions. J Hosp Med. 2015;10(12):811816. doi:10.1002/jhm.2434

4. Spevack R, Al Shukairi M, Jayaraman D, Dankoff J, Rudski L, Lipes J. Serial lung and IVC ultrasound in the assessment of congestive heart failure. Crit Ultrasound J. 2017;9(1):7. doi:10.1186/s13089-017-0062-3

5. Mantuani D, Frazee BW, Fahimi J, Nagdev A. Point-of-care multi-organ ultrasound improves diagnostic accuracy in adults presenting to the emergency department with acute dyspnea. West J Emerg Med. 2016;17(1):46-53.

6. Tierney DM, Huelster JS, Overgaard JD, et al. Comparative performance of pulmonary ultrasound, chest radiograph, and CT among patients with acute respiratory failure. Crit Care Med. 2020;48(2):151-157. doi:10.1097/CCM.0000000000004124

7. Mathews BK, Miller PE, Olson APJ. Point-of-care ultrasound improves shared diagnostic understanding between patients and providers. South Med J. 2018;111(7):395-400. doi:10.14423/ SMJ.0000000000000833

8. ACGME. ACGME Program Requirements for Graduate Medical Education in Internal Medicine. June 29, 2020. Accessed October 1, 2020. https://www.acgme.org/Portals/0/ PFAssets/ProgramRequirements/140_InternalMedicine_2020. pdf?ver=2020-06-29-161610-040

9. Schnobrich DJ, Gladding S, Olson AP, Duran-Nelson A. Pointof-care ultrasound in internal medicine: a national survey of educational leadership. J Grad Med Educ. 2013;5(3):498-502. doi:10.4300/JGME-D-12-00215.1

10. Reaume M, Siuba M, Wagner M, Woodwyk A, Melgar TA. Prevalence and Scope of point-of-care ultrasound education in internal medicine, pediatric, and medicine-pediatric residency programs in the United States. $J$ Ultrasound Med. 2019;38(6):1433-1439. doi:10.1002/jum.14821

11. CHEST. Critical Care Ultrasonography Certificate of Completion website. Accessed October 1, 2020. https://www.chestnet. org/Education/Advanced-Clinical-Training/Certificate-of-

Completion-Program/Critical-Care-Ultrasonography

12. Society of Hospital Medicine. Society of Hospital Medicine Point of Care Ultrasound Course website. Accessed October 1, 2020. https:/www.hospitalmedicine.org/clinical-topics/ultrasound/ pocus-certificate-of-completion/

13. Mathews BK, Reierson K, Vuong K, et al. The Design and evaluation of the Comprehensive Hospitalist Assessment and Mentorship with Portfolios (CHAMP) ultrasound program. $J$ Hosp Med. 2018;13(8):544-550. doi:10.12788/jhm.2938

14. Kelm DJ, Ratelle JT, Azeem N, et al. Longitudinal Ultrasound curriculum improves long-term retention among internal medicine residents. J Grad Med Educ. 2015;7(3):454-457. doi:10.4300/ JGME-14-00284.1

15. Mellor TE, Junga Z, Ordway S, et al. Not just hocus POCUS: Implementation of a point of care ultrasound curriculum for internal medicine trainees at a large residency program. Mil Med. 2019;184(11-12):901-906. doi:10.1093/milmed/usz124

16. Huang Y, Wang S, Liu Y, et al. A preliminary study on the ultrasonic manifestations of peripulmonary lesions of non-critical novel coronavirus pneumonia (COVID-19). SSRN. 2020;1-37. doi:10.21203/rs.2.24369/v1

17. Peng Q-Y, Wang X-T, Zhang L-N, Chinese Critical Care Ultrasound Study Group. Findings of lung ultrasonography of novel corona virus pneumonia during the 2019-2020 epidemic. Intensive Care Med. 2020;46(5):849-850. doi:10.1007/s00134020-05996-6

18. Mathews BK, Zwank M. Hospital medicine point of care ultrasound credentialing: an example protocol. J Hosp Med. 2017;12(9):767-772. doi:10.12788/jhm.2809 